\title{
Microbiota regulates bone marrow mesenchymal stem cell lineage differentiation and immunomodulation
}

\author{
E Xiao ${ }^{1,2 \dagger}$, Linhai He ${ }^{1,2 \dagger}$, Qiong Wu ${ }^{4,5}$, Junxiang Li, ${ }^{4,5}$, Yang He $e^{1,2}$, Lu Zhao ${ }^{1,2}$, Shuo Chen ${ }^{1,2}$, Jingang An ${ }^{1,2}$, \\ Yansong Liu ${ }^{6}$, Chider Chen ${ }^{3 *}$ and Yi Zhang ${ }^{1,2^{*}}$
}

\begin{abstract}
Health is dependent on the homeostasis of both inner and external microenvironments. The microbiota as the external microenvironment plays a critical role in regulation of several organ systems in mammals. However, it is unclear whether the microbiota regulates homeostasis of the skeletal system and bone marrow mesenchymal stem cells (BMMSCs). Here, using a well-established germ-free (GF) mouse model, we show that the microbiota significantly alters the stemness of BMMSCs in comparison to specific-pathogen-free (SPF)-derived BMMSCs. Colonization of GF mice with SPF microbiota (conventionalized (ConvD)) normalizes the proliferation and differentiation abilities of BMMSCs. On the other hand, normal microbiota is required to maintain immunomodulatory properties of BMMSCs through induction of activated T-cell apoptosis and cytokine secretion. GF-derived BMMSCs lose the capacity to ameliorate disease phenotypes in dextran sulfate sodium-induced experimental colitis mice. Mechanistically, single-cell RNA-sequencing analysis shows that ConvD BMMSCs have a similar gene expression pattern to SPF-derived BMMSCs, which have a distinct gene distribution from GF-derived BMMSCs.
\end{abstract}

\section{Background}

The mammal is inhabited by a vast number of bacteria, archaea, viruses, and eukaryotes. This microorganism coexistence with their hosts is referred to as the microbiota. It is reported that the human microbiota contains as many as $10^{14}$ bacterial cells, a number 10 times greater than the number of human cells [1]. The microbiota colonizes on the host mammal after they are exposed to the external environment. More than a billion years of mammalian-microbial coevolution has led to interdependency, resulting in a critical role of the microbiota in hematopoiesis [2], immune system development [3], neurologic signaling [4], host metabolism [5], and bone mass remodeling [6].

\footnotetext{
*Correspondence: chenc10@upenn.edu; zhangyi2000@263.net

${ }^{\dagger}$ Equal contributors

${ }^{3}$ Department of Anatomy and Cell Biology, University of Pennsylvania, School of Dental Medicine, Philadelphia, PA 19104, USA

'Department of Oral and Maxillofacial Surgery, Peking University School and Hospital of Stomatology, \#22 Zhongguancun South Avenue, Haidian District, Beijing 100081, China

Full list of author information is available at the end of the article
}

Bone marrow mesenchymal stem cells (BMMSCs), a kind of adult stromal cell in bone marrow, both contribute to the bone turnover [7] and form the unique bone marrow niche with hematopoietic stem cells [8]. BMMSCs show promising therapeutic potential based on their multipotent differentiation potential and immunomodulatory capacity $[9,10]$. However, whether these mesenchymal stem cells are "born with" these fantastic capacities or are educated by the microbiota was still not known. Thus, in this study we aimed to elucidate the effect of the microbiota on the multipotent differentiation and immunomodulatory abilities of BMMSCs.

\section{Results and discussion}

BMMSCs from germ-free mice exhibited higher colony forming ability and proliferation rate

To examine whether BMMSCs are regulated by the microbiota, BMMSCs were isolated from germ-free (GF) and specific-pathogen-free (SPF) mice. PCR analysis showed no bacteria detected in GF feces by universal bacteria primers, while conventionalized feces from GF mice colonized with SPF microbiota showed a similar 
pattern to the SPF group (Fig. 1d). Flow cytometry analysis showed that these two cell groups expressed similar mesenchymal cell surface markers CD73, CD90, CD105, CD166, and Sca1, while being negative for hematopoietic cell surface markers CD34 and CD45 (Fig. 1a). To examine the colony forming ability, 1 million BMMSCs were seeded in $60-\mathrm{mm}$ dishes to test the colony forming unit rate. Our data indicated that BMMSCs from GF mice formed significantly more colonies compared with those from the SPF mice (Fig. 1b). To further confirm the effects of the microbiota in BMMSC colony forming ability, GF mice were exposed in a conventional environment by cohousing with SPF mice for 2 weeks (conventionalized (ConvD)). The colony forming ability was significantly decreased to the level of SPF mice-derived BMMSCs (Fig. 1b). Next, using cell count kit 8 (CCK8), BMMSCs derived from GF mice also showed a higher proliferation rate when compared to SPF and ConvD BMMSCs (Fig. 1c). Besides, cell cycle analysis also showed more G2 and S-phase cell percentage in GFderived BMMSCs compared with that of SPF, and showed less Annexin V-positive cells in GF-derived
BMMSCs (Additional file 1: Figure S1D, E). Taken together, these data elucidate that the microbiota functionally controls BMMSC self-renewal capacities.

\section{Microbiota increases adipogenesis but decreases osteogenesis of BMMSCs}

Currently, there is broad intense interest in understanding the contribution of the microbiota to vertebrate/mammalian organ systems. Germ-free mice and antibiotic-treated mice have been shown to increase bone mineral density with reduction of osteoclasts and bone resorption $[6,11]$. As bone metabolism couples bone resorption with bone formation to maintain skeletal homeostasis, how the microbiota influences bone-forming cells, especially BMMSCs, is still largely unknown. To further examine the effect of the microbiota on BMMSC lineage commitment, we conducted adipogenic and osteogenic induction in vitro. Oil Red O staining showed that SPF-derived and ConvD-derived BMMSCs formed more Oil Red Opositive adipocytes than GF-derived BMMSCs (Fig. 2a). Western blot and real-time PCR analysis further confirmed that the key adipogenic transcription factors
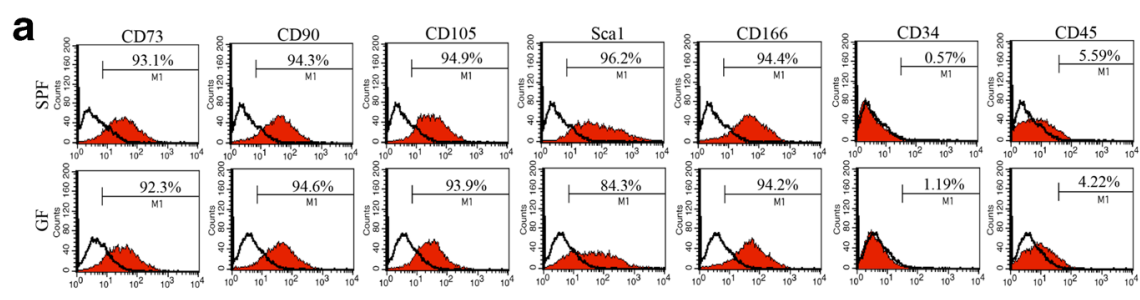

b
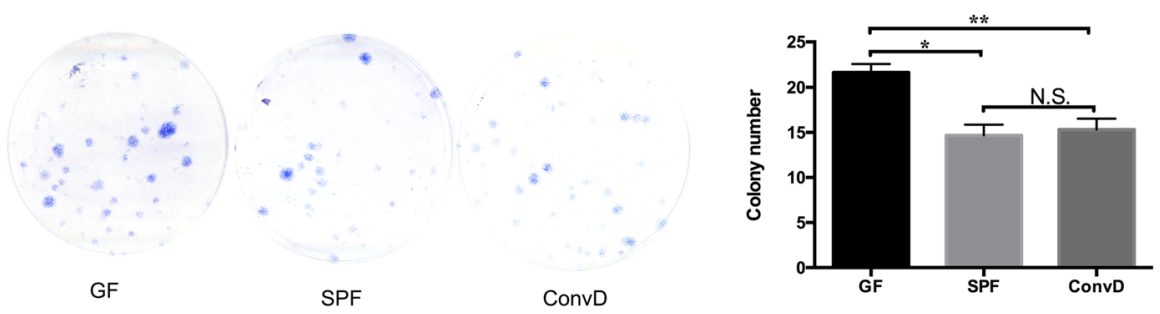

C
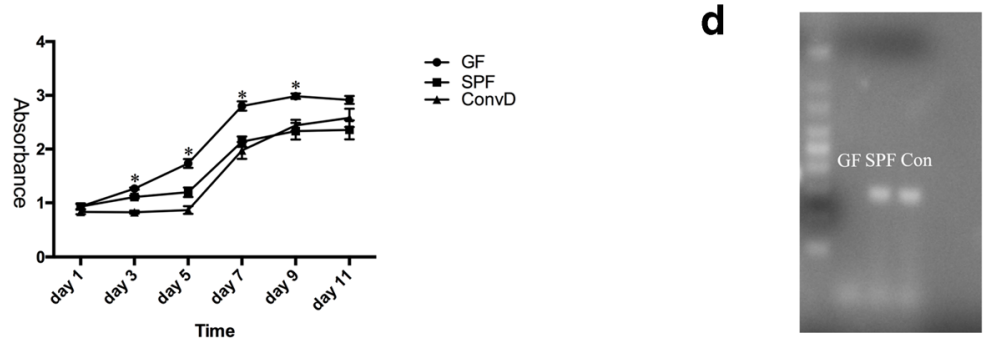

Fig. 1 Characterization and proliferation of GF, SPF, and ConvD BMMSCs. a Flow cytometry analysis shows that BMMSCs derived from SPF and GF mice had similar positive markers (CD73, CD90, CD105, Sca1, and CD166) and negative markers (CD34 and CD45). b Colony forming unit experiments show BMMSCs derived from GF mice had higher colony forming rate compared to that from SPF and ConvD mice (21.67 \pm 0.88 vs $14.67 \pm 1.20$ and $15.33 \pm 1.2)$. c CCK8 analysis shows BMMSCs from GF mice had higher proliferation capacity compared to that from SPF and ConvD mice. $\mathbf{d}$ PCR analysis shows that no bacterium was detected in GF feces using universal bacteria primers 515F and 806R. All experimental data verified in at least three independent experiments. Error bars represent the SEM from the mean values. ${ }^{*} P<0.001 ;{ }^{*} P<0.05$. ConvD conventionalized, GF germ free, SPF specific pathogen free, N.S. not significant 


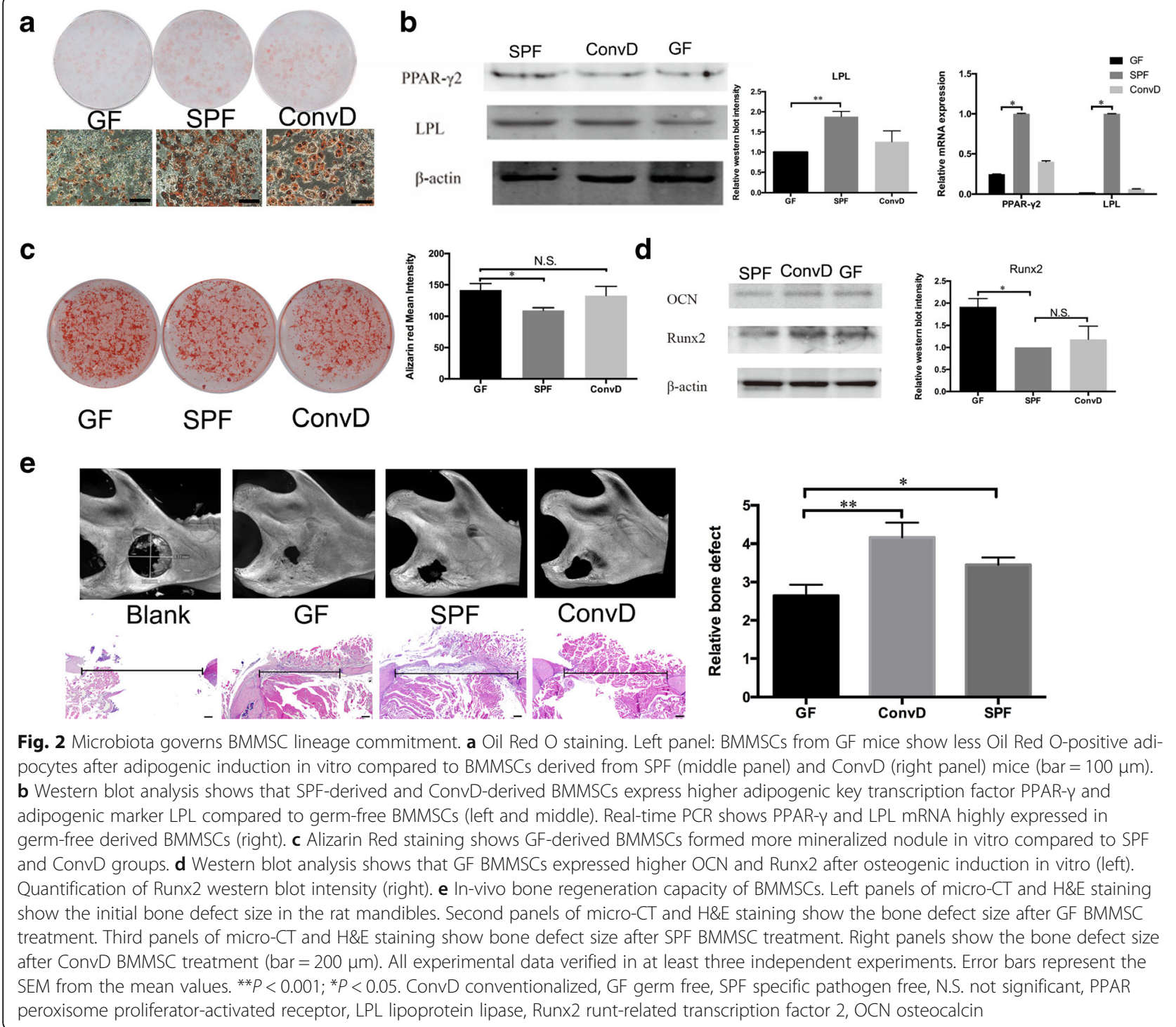

peroxisome proliferator-activated receptor $\gamma$ (PPAR- $\gamma 2$ ) and adipogenic marker lipoprotein lipase (LPL) were highly expressed in the BMMSCs from the SPF and ConvD groups after adipogenic induction, while GFderived BMMSCs expressed significantly lower PPARy and LPL (Fig. 2b). On the other hand, osteogenic study showed that BMMSCs from GF mice formed more mineralization deposit than SPF/ConvD BMMSCs, which was detected by Alizarin Red staining (Fig. 2c). Western blot analysis indicated that the expression levels of osteogenic transcription factor runt-related transcription factor 2 (Runx2) and osteogenic marker osteocalcin (OCN) were significantly higher in GF-derived BMMSCs when compared with SPF/ConvD BMMSCs (Fig. 2d). These data demonstrated that the microbiota significantly altered BMMSC lineage differentiation in vitro. To test the in-vivo bone formation abilities, we implanted GF-derived, SPF- derived, and ConvD-derived BMMSCs in a mandibular bone defect model to regenerate bone tissue. Micro-CT analysis showed that GF-derived BMMSCs greatly repaired mandibular bone defect, while BMMSCs from the SPF and ConvD groups showed less bone regeneration with bigger size bone defect remaining (Fig. 2e). In addition, hematoxylin and eosin (H\&E) staining also showed significantly more bone regeneration in the GF BMMSC implanted group when compared with the SPF and ConvD BMMSC implanted groups (Fig. 2e). To examine whether the superior osteogenesis ability of GF BMMSCs can contribute to bone mineral density (BMD) in vivo, we performed micro-CT analysis to show that femoral BMD of GF mice was significantly increased compared to SFP mice (Additional file 1: Figure S1A, B). After 2 weeks of cohousing, the ConvD femoral BMD was largely decreased to the level of SPF mice (Additional file 1: Figure S1A, B). 
Collectively, these findings indicated that the microbiota inhibited BMMSC proliferation and adipogenesis but increased osteogenesis and in-vivo regenerative abilities. Interestingly, the microbiota increases adipogenic differentiation, which is consistent with reduced obesity in germ-free mice [12]. Probiotic and prebiotic treatments are able to increase bone mass and BMD, indicating that intestinal microbiota may impact bone metabolism and health maintenance [13-16]. These findings connect gut microbiota and skeletal remodeling, which prompts us to investigate the impact of the microbiota on BMMSCs and bone tissue regeneration. They also suggest that physiological regulation of the osteoblastic/adipogenic lineage switch in the bone compartment involves the microbiota.
BMMSCs from GF mice were deficient in immunomodulation Next, we asked whether the microbiota can affect BMMSC immunomodulation. It has been reported that BMMSCs cannot suppress immune reactions, unless they were preactivated by certain combinations of the inflammatory environment [17, 18]. The microbiota played a critical role in the maturation of immune system and tolerance $[19,20]$. Thus, we reasoned that the immunomodulatory capacities of BMMSCs may be affected by the microbiota. We first cocultured BMMSCs from different groups with pan-T cells and examined their abilities to induce T-cell apoptosis. Flow cytometry analysis showed that SPF-derived and ConvDderived BMMSCs significantly induced more T-cell apoptosis when compared with BMMSCs from germfree mice (Fig. 3a). In addition, cytokine array analysis

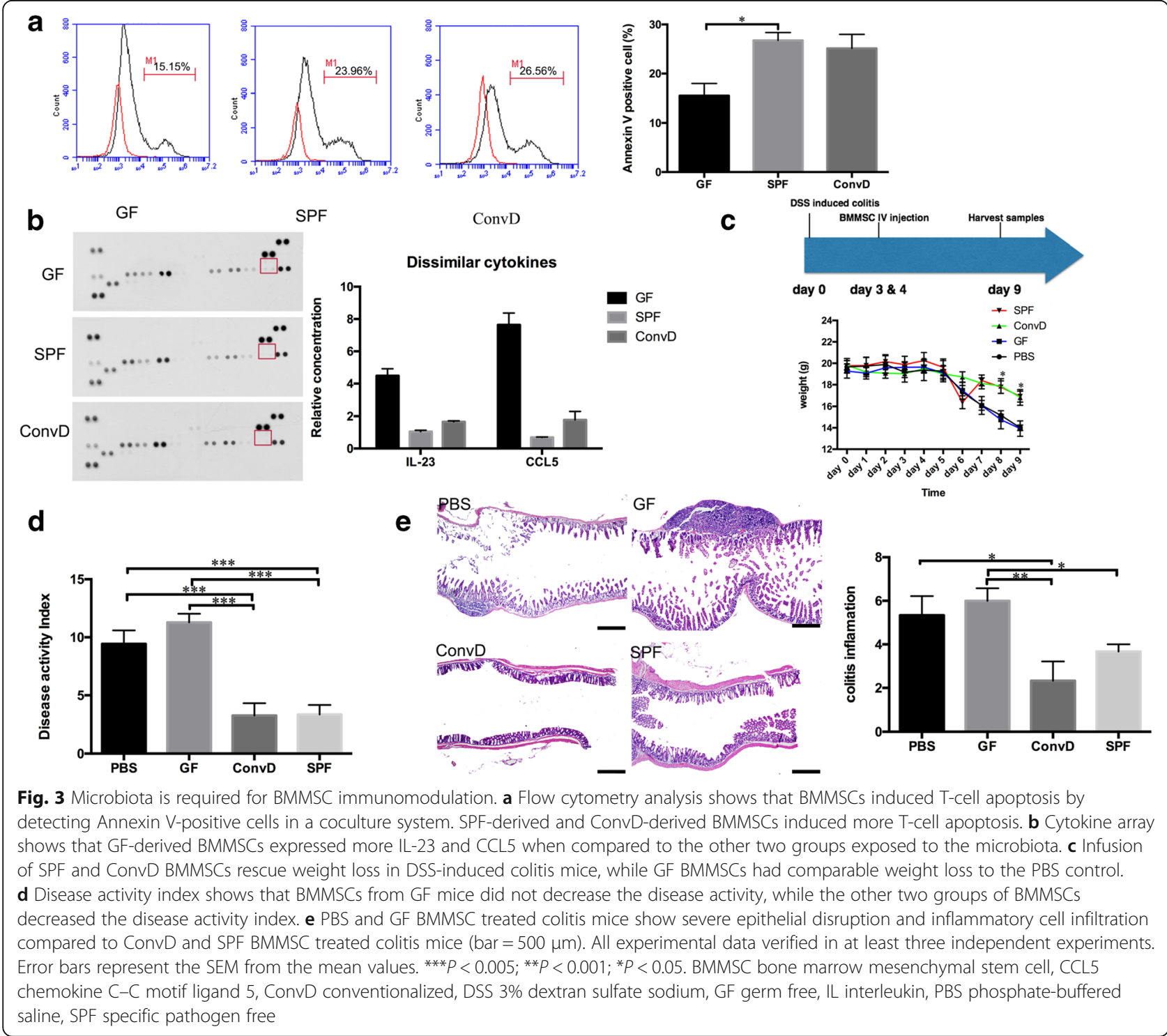


showed that GF-derived BMMSCs secreted increased proinflammatory factors interleukin-23 (IL-23) and chemokine $\mathrm{C}-\mathrm{C}$ motif ligand 5 (CCL5) (Fig. 3b) which contributed to inflammatory disease. To further investigate the ability for immunomodulation of BMMSCs, the GF, SPF, and ConvD BMMSCs were systemically infused into a $3 \%$ dextran sulfate sodium (DSS)-induced experimental colitis mouse model [21] at 3 days post DSS induction (Fig. 3c). The colitis mice had significantly reduced body weight compared to C57BL6 control mice from days 5 to 9 post DSS induction (Fig. 3c). After infusion of either SPF-derived or ConvD-derived BMMSCs, but not GF BMMSCs, the body weight of the colitis mice was partially rescued at 9 days post DSS induction (Fig. 3c). The disease activity index (DAI), including body weight loss, diarrhea, and bleeding, was significantly elevated in the colitis mice compared to the control group. After infusion of SPF and ConvD BMMSCs, the DAI in the colitis mice was obviously decreased at day 9, while GF BMMSC infusion failed to reduce the DAI (Fig. 3d). BMMSCs from microorganism-exposed mice such as SPF and ConvD significantly relieved the disease activity more than twofold compared to the GF BMMSC and PBS treated groups $(3.36 \pm 0.48,3.28 \pm 0.61$ vs $11.29 \pm 0.43$, $9.43 \pm 0.67$; Fig. $3 d$ ). In addition, H\&E staining and the quantitative inflammatory score showed that the SPFderived and ConvD-derived BMMSCs greatly decreased the colitis inflammation (inflammation scores $3.67 \pm 0.33$ and $2.33 \pm 0.88$ ) compared with PBS and GF BMMSC treated groups (inflammation scores $6.0 \pm$ 0.58 and $5.33 \pm 0.88$; Fig. 3e).

The role of the microbiota in regulating immune cell polarization and human diseases is receiving increasing attention [22, 23]. BMMSCs reside in the skeleton to maintain osteoblastic lineage cell function and serve as a niche for hematopoietic stem cells, which involves several physiological regulations and interplays with the immune system. Our data indicated that in the unique niche of both mesenchymal stem cells and hematopoietic stem cells, the microbiota also regulated the BMMSCs, which may be related to the maturation or apoptosis of hematopoietic cell lines.

\section{Single-cell RNA-sequencing analysis identified three pathway categories regulated by microbiota}

To investigate and compare the gene expression differences between GF, SPF, and ConvD BMMSCs, 35 single ConvD-derived BMMSCs, 28 single GF BMMSCs, and 32 SPF BMMSCs were isolated and sequenced. Principal component analysis showed that almost all SPF group cells cluster together and most of the ConvD group cells distributed together and closed to the SPF group cells (Fig. 4a). BMMSCs from GF mice were separated into two parts, in which 19 germ-free BMMSCs were clustered with SPF and GF group cells, while nine GF BMMSCs showed a dramatically different expression pattern (Fig. 4a). These subcluster populations may cause the different behaviors of GF BMMSCs in both self-renewal and lineage differentiation. Correlation analysis further confirmed the higher correlation between most of the SPF-derived and ConvD-derived BMMSCs (Fig. 4b), indicating that the microbiota homogenized the gene expression of BMMSCs in SPF and ConvD mice. In contrast, BMMSCs from germ-free mice contained subpopulation cells that kept the distinct expression pattern when compared with microbiota-educated BMMSCs. Single-cell RNA-sequencing data showed that there were totally 189 dissimilar gene expression between germ-free BMMSC subpopulations and microbiotaeducated cells (Fig. 4b). These differentially expressed genes belonged to several pathways (Fig. 4c), and could be concluded in three major categories, including cell metabolic pathways, HIF-1/inflammatory signaling, and neurodegenerative pathways (Additional file 2: Table S1). Metabolic pathways, such as ribosome, glycolysis, amino acid biosynthesis, carbon metabolism, and oxidative phosphorylation, have been shown to play an important role in regulating BMMSC proliferation and differentiation [24-26]. In addition, HIF-1 and several infection/inflammatory signaling pathways may be involved in cytokine and chemokine secretion of BMMSCs, which is critical for BMMSC immunomodulation. Remarkably, in the different gene interaction map, microbiota-educated BMMSCs showed significantly elevated chemokines and interleukin-10 (IL-10) expression, which was also related to the capacities of immunomodulation in BMMSCs (Fig. 4d).

The microbiota is involved in the regulation of multiple host metabolic pathways, which activates immuneinflammatory axes and signaling pathways [27]. As the bone compartment is unlikely to directly contact with microbes, it is easy to envision that the microbiome could influence BMMSCs through regulating metabolic pathways. Our single-cell RNA-sequencing data further confirm that several major metabolic pathways are significantly different between GF and SPF/ConvD BMMSCs, implying metabolism may connect the microbiota and BMMSCs to maintain bone homeostasis. Besides, our results show that HIF-1 signaling may be the major regulator in BMMSC immunomodulation, since HIF-1 has been reported to crosstalk with inflammatory transcription factor $\mathrm{NF}_{\kappa} \mathrm{B}$ and regulates release of cytokines and chemokines to control immune response $[28,29]$. In summary, this is the first study to link the microbiota with BMMSC function, and single- 
a

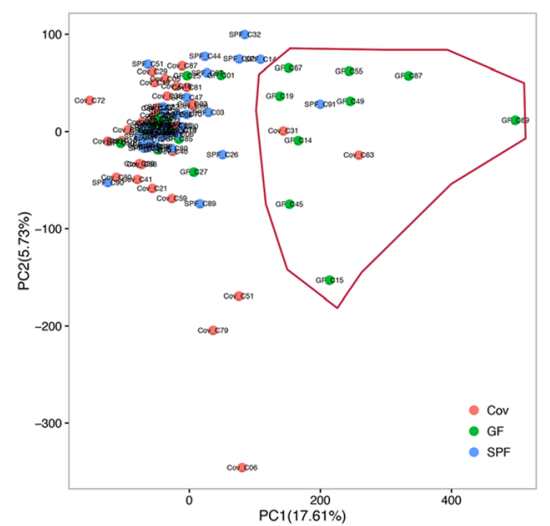

b

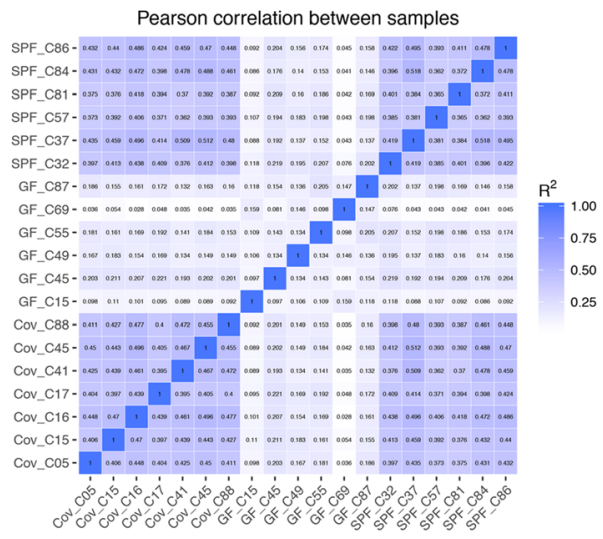
SPF_C $37-0$ ond on (0)

C

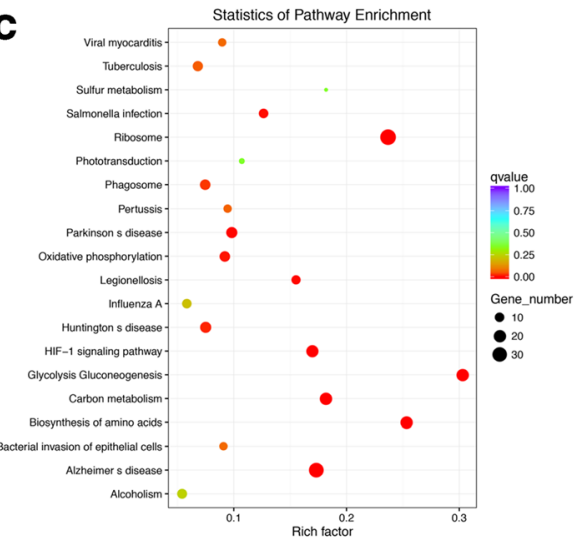

d

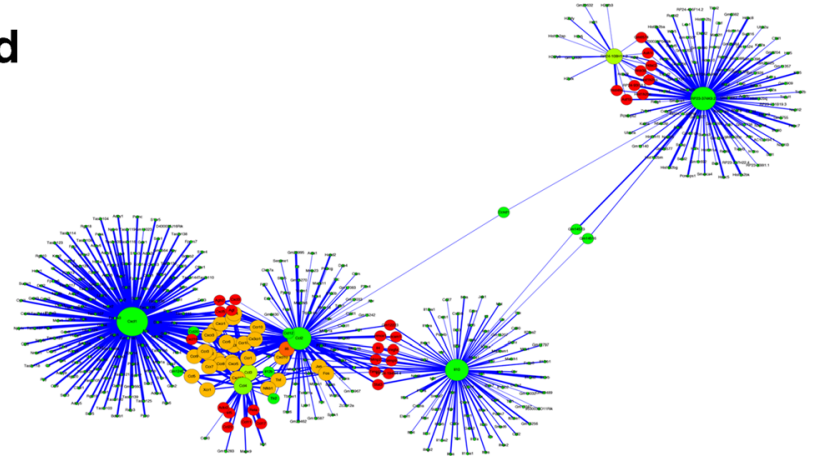

Fig. 4 Single-cell RNA-sequencing identifies differences of three major pathway categories between GF and SPF/ConvD BMMSCs. a Unsupervised cell population clustering shows SPF and ConvD BMMSCs clustered together, while GF BMMSCs distributed into two parts. b Correlation analysis shows most mRNA expression in SPF and ConvD BMMSCs was related, distinct from GF BMMSCs. c Pathway analysis shows the different expressing genes between GF and SPF/ConvD BMMSCs. d Different gene-related protein interaction networks show that chemokines such as CXCL1, CCL2, and IL10 play a central role in BMMSC immunomodulation. GF germ free, SPF specific pathogen free

cell RNA-sequencing analysis further provides detailed pathway prediction to connect the microbiota to regulating BMMSCs and bone metabolism.

In conclusion, we have revealed that the microbiota alters the differentiation potential and enhances the immunomodulation capacity of BMMSCs. This study provides a new point of view on how BMMSCs gain their therapeutic function.

\section{Additional files}

Additional file 1: Figure S1. Showing comparisons of bone mineral density, bone morphology parameters, cell cycle analysis, and apoptosis cell percentages between the SPF group and the GF group. (PDF $1127 \mathrm{~kb}$ )

Additional file 2: Table S1. Presenting pathway enrichment analysis. (DOCX $12 \mathrm{~kb})$

Additional file 3: Supplemental materials and methods used in this study. (DOCX $24 \mathrm{~kb}$ ) 


\section{Acknowledgements}

The authors thank Ting Zhang from Department of Orthodontics, Peking University School and Hospital of Stomatology for her help in mouse BMMSC culture

\section{Funding}

This work was supported by grants from National Natural Science Foundation of China (No. 81500819 and No. 81371117) and National Institute of Dental and Craniofacial Research, National Institutes of Health, Department of Health and Human Services (K99E025915 to CC).

\section{Availability of data and materials}

All data generated or analyzed during this study are included in this published article.

All methods and materials used in this study are listed in Additional file 3.

\section{Authors' contributions}

EX contributed to conception, design, and data acquisition. LHH contributed to data acquisition and analysis. QW and JXL contributed to single-cell sequencing and data analysis. HY, ZL, CS, and JGA contributed to the data collection, analysis, and interpretation. YSL contributed to raising mice and mice sample harvest. CC contributed to conception, design, and data acquisition and drafted the manuscript. YZ contributed to conception and design, and critically revised the manuscript. All authors read and approved the final manuscript.

\section{Ethics approval}

All animal experiments were approved by The Ethics Committee of the Peking University Health Science Center (LA2016149).

\section{Consent for publication}

Not applicable.

\section{Competing interests}

The authors declare that they have no competing interests.

\section{Publisher's Note}

Springer Nature remains neutral with regard to jurisdictional claims in published maps and institutional affiliations.

\section{Author details}

${ }^{1}$ Department of Oral and Maxillofacial Surgery, Peking University School and Hospital of Stomatology, \#22 Zhongguancun South Avenue, Haidian District, Beijing 100081, China. ${ }^{2}$ National Engineering Laboratory for Digital and Material Technology of Stomatology, Beijing Key Laboratory of Digital Stomatology, \#22 Zhongguancun South Avenue, Beijing 100081, China. ${ }^{3}$ Department of Anatomy and Cell Biology, University of Pennsylvania, School of Dental Medicine, Philadelphia, PA 19104, USA. ${ }^{4}$ MOE Key Laboratory of Bioinformatics, Center for Synthetic and System Biology, Tsinghua University, Beijing, China. ${ }^{5}$ School of Life Sciences, Tsinghua University, Beijing 100084, China. ${ }^{6}$ Chinese Academy of Sciences Shanghai Laboratory Animal Center, Songjiang District, Shanghai 201615, China.

Received: 2 July 2017 Revised: 24 August 2017

Accepted: 12 September 2017 Published online: 29 September 2017

\section{References}

1. Ley RE, Peterson DA, Gordon Jl. Ecological and evolutionary forces shaping microbial diversity in the human intestine. Cell. 2006;124(4):837-48.

2. Khosravi A, Yanez A, Price JG, Chow A, Merad M, Goodridge HS, Mazmanian SK. Gut microbiota promote hematopoiesis to control bacterial infection. Cell Host Microbe. 2014;15(3):374-81.

3. Macpherson AJ, Harris NL. Interactions between commensal intestinal bacteria and the immune system. Nat Rev Immunol. 2004;4(6):478-85.

4. Yano JM, Yu K, Donaldson GP, Shastri GG, Ann P, Ma L, Nagler CR, Ismagilov RF, Mazmanian SK, Hsiao EY. Indigenous bacteria from the gut microbiota regulate host serotonin biosynthesis. Cell. 2015;161(2):264-76.

5. Canfora EE, Jocken JW, Blaak EE. Short-chain fatty acids in control of body weight and insulin sensitivity. Nat Rev Endocrinol. 2015;11(10):577-91.
6. Cho I, Yamanishi S, Cox L, Methe BA, Zavadil J, Li K, Gao Z, Mahana D, Raju $\mathrm{K}$, Teitler I, et al. Antibiotics in early life alter the murine colonic microbiome and adiposity. Nature. 2012;488(7413):621-6.

7. Zhou BO, Yue R, Murphy MM, Peyer JG, Morrison SJ. Leptin-receptorexpressing mesenchymal stromal cells represent the main source of bone formed by adult bone marrow. Cell Stem Cell. 2014;15(2):154-68.

8. Mendez-Ferrer S, Michurina TV, Ferraro F, Mazloom AR, Macarthur BD, Lira SA, Scadden DT, Ma'ayan A, Enikolopov GN, Frenette PS. Mesenchymal and haematopoietic stem cells form a unique bone marrow niche. Nature. 2010; 466(7308):829-34.

9. Prockop DJ. Marrow stromal cells as stem cells for nonhematopoietic tissues. Science. 1997;276(5309):71-4.

10. Uccelli A, Pistoia V, Moretta L. Mesenchymal stem cells: a new strategy for immunosuppression? Trends Immunol. 2007;28(5):219-26.

11. Sjogren $K$, Engdahl $C$, Henning P, Lerner UH, Tremaroli V, Lagerquist MK, Backhed $\mathrm{F}$, Ohlsson $\mathrm{C}$. The gut microbiota regulates bone mass in mice. J Bone Mineral Res. 2012;27(6):1357-67.

12. Suarez-Zamorano N, Fabbiano S, Chevalier C, Stojanovic O, Colin DJ, Stevanovic A, Veyrat-Durebex C, Tarallo V, Rigo D, Germain S, et al. Microbiota depletion promotes browning of white adipose tissue and reduces obesity. Nat Med. 2015;21(12):1497-501.

13. Britton RA, Irwin R, Quach D, Schaefer L, Zhang J, Lee T, Parameswaran N, McCabe LR. Probiotic $L$. reuteri treatment prevents bone loss in a menopausal ovariectomized mouse model. J Cell Physiol. 2014;229(11):1822-30.

14. McCabe LR, Irwin R, Schaefer $L$, Britton RA. Probiotic use decreases intestinal inflammation and increases bone density in healthy male but not female mice. J Cell Physiol. 2013;228(8):1793-8.

15. Scholz-Ahrens KE, Schrezenmeir J. Inulin and oligofructose and mineral metabolism: the evidence from animal trials. J Nutr. 2007;137(11 Suppl): 2513S-23S.

16. Yang $L C, W u J B, L u T J$, Lin WC. The prebiotic effect of Anoectochilus formosanus and its consequences on bone health. Br J Nutr. 2013;109(10): 1779-88.

17. Ren G, Zhang L, Zhao X, Xu G, Zhang Y, Roberts Al, Zhao RC, Shi Y. Mesenchymal stem cell-mediated immunosuppression occurs via concerted action of chemokines and nitric oxide. Cell Stem Cell. 2008;2(2):141-50.

18. Ren G, Su J, Zhang L, Zhao X, Ling W, L'Huillie A, Zhang J, Lu Y, Roberts Al, $\mathrm{Ji} W$, et al. Species variation in the mechanisms of mesenchymal stem cellmediated immunosuppression. Stem Cells. 2009;27(8):1954-62.

19. Sommer F, Backhed F. The gut microbiota-masters of host development and physiology. Nat Rev Microbiol. 2013;11(4):227-38.

20. Sekirov I, Russell SL, Antunes LC, Finlay BB. Gut microbiota in health and disease. Physiol Rev. 2010;90(3):859-904

21. Alex P, Zachos NC, Nguyen T, Gonzales L, Chen TE, Conklin LS, Centola M, Li $X$. Distinct cytokine patterns identified from multiplex profiles of murine DSS and TNBS-induced colitis. Inflamm Bowel Dis. 2009:15(3):341-52.

22. Backhed F, Fraser CM, Ringel Y, Sanders ME, Sartor RB, Sherman PM, Versalovic J, Young V, Finlay BB. Defining a healthy human gut microbiome: current concepts, future directions, and clinical applications. Cell Host Microbe. 2012;12(5):611-22.

23. Lemon KP, Armitage GC, Relman DA, Fischbach MA. Microbiota-targeted therapies: an ecological perspective. Sci Transl Med. 2012;4(137):137rv135.

24. Pattappa G, Heywood HK, de Bruijn JD, Lee DA. The metabolism of human mesenchymal stem cells during proliferation and differentiation. J Cell Physiol. 2011;226(10):2562-70.

25. Tormos KV, Anso E, Hamanaka RB, Eisenbart J, Joseph J, Kalyanaraman B, Chandel NS. Mitochondrial complex III ROS regulate adipocyte differentiation. Cell Metab. 2011;14(4):537-44.

26. Shum LC, White NS, Mills BN, Bentley KL, Eliseev RA. Energy metabolism in mesenchymal stem cells during osteogenic differentiation. Stem Cells Dev. 2016;25(2):114-22.

27. Nicholson JK, Holmes E, Kinross J, Burcelin R, Gibson G, Jia W, Pettersson S Host-gut microbiota metabolic interactions. Science. 2012;336(6086):1262-7.

28. Walmsley SR, Print C, Farahi N, Peyssonnaux C, Johnson RS, Cramer T, Sobolewski A, Condliffe AM, Cowburn AS, Johnson N, et al. Hypoxiainduced neutrophil survival is mediated by HIF-1alpha-dependent NFkappaB activity. J Exp Med. 2005;201(1):105-15.

29. Scortegagna M, Cataisson C, Martin RJ, Hicklin DJ, Schreiber RD, Yuspa SH, Arbeit JM. HIF-1alpha regulates epithelial inflammation by cell autonomous NFkappaB activation and paracrine stromal remodeling. Blood. 2008;111(7):3343-54. 